\title{
Effects of atorvastatin combined with low-molecular-weight heparin on plasma inflammatory cytokine level and pulmonary pathophysiology of rats with sepsis
}

\author{
FEI JING ${ }^{1}$, MING LI $^{2}$, HONGSHENG REN ${ }^{3}$, JITIAN ZHANG ${ }^{4}$, QINGCHUN YAO ${ }^{3}$, \\ YUFENG $\mathrm{CHU}^{3}$ and $\mathrm{CHUNTING}$ WANG $^{3}$
}

\begin{abstract}
Departments of ${ }^{1}$ Endocrinology and Metabolism, ${ }^{2}$ Rheumatology and Clinical Immunology, ${ }^{3}$ Intensive Care Unit, and ${ }^{4}$ Nutrition, Shandong Provincial Hospital Affiliated to Shandong University, Jinan, Shandong 250021, P.R. China
\end{abstract}

Received February 15, 2016; Accepted May 20, 2016

DOI: $10.3892 /$ etm.2016.3372

\begin{abstract}
The aim of the present study was to investigate the effect of atorvastatin combined with low-molecular-weight heparin $(\mathrm{LMWH})$ on plasma early inflammatory cytokine levels as well as pulmonary pathophysiology of rats with sepsis. A total of 122 rats were randomly divided into five groups including the sham operation group ( $\mathrm{n}=10)$, CLP group $(\mathrm{n}=10)$, atorvastatin group ( $\mathrm{n}=34,20 \mathrm{mg} / \mathrm{kg} / \mathrm{day}), \mathrm{LMWH}$ group $(\mathrm{n}=34$, $100 \mathrm{IU} / \mathrm{kg} / \mathrm{day}$ ), and atorvastatin combined with LMWH group $(\mathrm{n}=34)$. Blood samples from 6 rats in each group were collected to detect TNF- $\alpha$, IL-1 $\beta$ and HMGB1 concentration in plasma by linked immunosorbent assay at baseline and postoperatively at 4, 8, 12 and 24 h. Pulmonary pathophysiology was observed postoperatively at $24 \mathrm{~h}$. The remaining 10 rats in each group were used to calculate the 7-day cumulative mortality rate. Compared to the sham operation group, the scores in CLP were greater than those of the sham operation group $(\mathrm{P}<0.05)$. Compared to the CLP group, the sepsis severity scores of the atorvastatin, $\mathrm{LMWH}$, and atorvastatin combined with LMWH groups decreased gradually. Significant difference was detected in the four groups $(\mathrm{P}<0.050 .01)$. Compared to the sham operation group, at 4, 8, 12 and $24 \mathrm{~h}$, the TNF- $\alpha$, IL-1 $\beta$ and HMGB1 levels in plasma in CLP increased significantly $(\mathrm{P}<0.01)$. Compared to the CLP group, the TNF- $\alpha$, IL- $1 \beta$ and HMGB1 levels of plasma in other groups decreased gradually, and there was a significant difference in the four groups $(\mathrm{P}<0.01)$. At $24 \mathrm{~h}$ post operation, compared to the sham operation group, the damage of pulmonary pathophysiology in CLP was more severe. Compared to the CLP group, the damage of pulmonary
\end{abstract}

Correspondence to: Dr Hongsheng Ren, Department of Intensive Care Unit, Shandong Provincial Hospital Affiliated to Shandong University, 324 Jingwu Road, Jinan, Shandong 250021, P.R. China E-mail: hongsheng-ren@163.com

Key words: sepsis rats, cecal ligation and puncture, atorvastatin, low-molecular-weight heparin, sepsis severity, inflammatory cytokines, pulmonary pathophysiology, cumulative mortality rate pathophysiology in other groups was slight. Compared to the CLP group, the 7-day cumulative mortality rate in other groups decreased significantly $(\mathrm{P}<0.05)$. In conclusion, atorvastatin, combined with LMWH can decrease sepsis severity, plasma inflammatory cytokine levels, pulmonary pathophysiology, and the 7-day cumulative mortality rate. Atorvastatin, and LMWH may therefore be useful for the treatment of sepsis due to its ability to inhibit the release of TNF- $\alpha$, IL- $1 \beta$ and HMGB1 in septic rats.

\section{Introduction}

Sepsis is a common complication of severe trauma, large area burns, severe shock, serious infection and surgery. Sepsis can progress to septic shock, acute respiratory distress syndrome (ARDS), acute kidney injury (AKI), and multiple organ dysfunction syndrome (MODS), which is one of the major causes of mortality in critically ill patients (1). However, whether sepsis causes mortality is mainly determined by the release intensity of inflammatory factors and the response to inflammation (2). Sepsis bundle therapy is considered the main treatment method, including removing etiological factors, fluid resuscitation, use of vasoactive drugs, effective antibiotic use, immune regulation and hemofiltration (3). However, the mortality rate of severe sepsis or septic shock remains high, and ranges $30-50 \%$ (4).

Previous studies suggested that the pathogenesis of sepsis involves inflammation, coagulation and immune reactivity (5). Sepsis is a serious stage of bacterial infection, which involves the release of many types of inflammatory factors, such as tumor necrosis factor- $\alpha$ (TNF- $\alpha)(6)$, interleukin-1 $\beta$ (IL-1 $\beta)$ (7), IL-6 (8) and high mobility group protein box 1 (HMGB1) (9). Statins are widely used in the regulation of lipid metabolism. Low-molecular-weight heparin (LMWH) is widely used as an anticoagulant drug. Previous findings showed that statins are beneficial to patients with sepsis and LMWH improves the prognosis of patients with sepsis $(9,10)$. However, the mechanism and efficacy of statins combined with LMWH in the treatment of sepsis has not been reported.

In the present study, the rats underwent cecal ligation and puncture (CLP). We evaluated the effect of atorvastatin 
combined with LMWH on early and late inflammatory plasma cytokine levels, pulmonary pathophysiology and the cumulative mortality rate of rats with sepsis, to clarify the mechanism of statins combined with LMWH in the inflammatory process of sepsis. Thus, the results provide a theoretical basis for the clinical treatment of sepsis with combined statins and LMWH.

\section{Materials and methods}

Equipment. The DS-88 electronic scale was purchased from Wuhan Automation Instrument Factory (Wuhan, China). The high-speed refrigerated centrifuge was purchased from Eppendorf AG (Hamburg, Germany) and Beckman Coulter, Inc.(Brea, CA, USA). The SHH WZI600S digital display - three electric thermostatic water bath box was purchased from the Shanghai Medical Equipment factory (Shanghai, China). The Versa Max MR96A enzyme mark instrument was purchased from Shenzhen Mindray Bio-Medical Electronics Co., Ltd. (Shenzhen, China). NanoDrop 2000c ultraviolet spectrophotometer was purchased from Thermo Fisher Scientific, Inc. (Wilmington, DE, USA). The inverted microscope was purchased from Olympus (Tokyo, Japan) and the light microscope was purchased from Carl Zeiss AG A2 (Oberkochen, Germany).

Materials and reagents. Atorvastatin (Lipitor), $20 \mathrm{mg} / \mathrm{plate}$ was purchased from Pfizer, Inc. (Dalian, China), national drug approval no. J20130129. LMWH (Fraxiparine), 0.4 ml/tube, was purchased from Sanofi Winthrop Industrie SA (Paris, France), approval no. H20140349. The anesthetic purchased was $3 \%$ pentobarbital sodium. Surgical supplies included Ethicon braided non-absorbable sutures (black), 15x60 cm, purchased from Johnson \& Johnson (Somerville, NJ, USA). The KGERC102a KeyGen rat, TNF- $\alpha$, and IL-1 $\beta$ enzymelinked immunosorbent assay (ELISA) kit, were purchased from Nanjing KeyGen Biotech Co., Ltd. (Nanjing, China). The HMGB1 ELISA kit was purchased from Beijing Wantai Biological Pharmaceutical Co., Ltd. (Beijing, China).

Animal groupings and treatment. In total, 122 healthy male Sprague-Dawley rats weighing 178-235 g, were procured from the Laboratory Animal Center of Beijing Huafu Medical-Technological Co. (animal batch no. scxk2014-0004). The rats were housed at $20-26^{\circ} \mathrm{C}$, maintained in $40-70 \%$ air humidity and with a natural length of day and night cycle in the laboratory. Five rats were placed in each cage $(500 \times 300 \times 200 \mathrm{~mm})$ with free activity and free access to complete formula feed (Jinan Xingkang Biotechnology Co., Ltd., Jinan, China) and water. The rats were divided randomly into five groups, including the sham operation group $(n=10)$, CLP group $(n=10)$, atorvastatin group $(n=34)$, LMWH group $(n=34)$. The study was approved by the ethics committee of Shandong University (Shandong, China).

For the sham operation group, rats were administered starch solution (40 mg/kg.day dissolved in $2 \mathrm{ml} 0.9 \% \mathrm{NaCl}$ ). The suspension was given through intragastric infusion for 5 days. The rats were fasted $12 \mathrm{~h}$ prior to surgery. The rats were anesthetized by injection of $3 \%$ pentobarbital sodium (45 mg/kg body weight, intraperitoneally) and a sterile $2-\mathrm{cm}$ ventral midline incision was performed. The cecum was disturbed and returned to the abdomen. The abdominal incision was closed in layers. The rats were then housed with free activity and feed.

The CLP rats underwent the same intragastric infusion procedure as described above prior to surgery. The rat model of sepsis was performed by CLP (12).

For the atorvastatin group, atorvastatin was ground with a mortar. Atorvastatin suspensions were prepared $(20 \mathrm{mg} / \mathrm{kg} \cdot$ day of atorvastatin dissolved in $2 \mathrm{ml} 0.9 \% \mathrm{NaCl}$ ). The suspension was given through intragastric infusion for 5 days. The rat model of sepsis was then performed by CLP (11).

The LMWH rats were administered starch suspension (40 $\mathrm{mg} / \mathrm{kg} \cdot \mathrm{d}$ dissolved in $2 \mathrm{ml} 0.9 \% \mathrm{NaCl}$ ). The suspension was given through intragastric infusion for 5 days. LMWH heparin was injected by hypodermic needle (100 IU/ $\mathrm{kg} \cdot$ day) for 5 days. The rat model of sepsis was performed by CLP (11).

For the atorvastatin combined with LMWH group, atorvastatin suspensions were prepared $(20 \mathrm{mg} / \mathrm{kg} \cdot$ day of atorvastatin dissolved in $2 \mathrm{ml} 0.9 \% \mathrm{NaCl}$ ). The suspension was given through intragastric infusion, and LMWH was injected using a hyperdermic needle (100 IU/ $\mathrm{kg} \cdot$ day) for 5 days. The rat models of sepsis was performed by CLP.

CLP rats were fasted $12 \mathrm{~h}$ before surgery (12). The rats were anesthetized by injection of $3 \%$ pentobarbital sodium (45 mg/kg body weight, intraperitoneally) and a sterile $2-\mathrm{cm}$ ventral midline incision was performed. The cecum was exposed and ligated with $30 \%$ nitrile silk, the intestinal mesenteric vessel was avoided while ligating, the cecum was punctured twice ( $3 \mathrm{~mm}$ region between them) with a 9-gauge needle. The abdominal incision was closed in layers. Normal saline ( $3 \mathrm{ml} / 100 \mathrm{~g}$ body weight) was given subcutaneously immediately to prevent dehydration.

Sepsis severity assessment standards. The severity of sepsis of the animals was scored according to modified sepsis severity assessment standards (Table I).

Specimen collection and detection. Before and after surgery and 4, 8, 12 and $24 \mathrm{~h}$ after surgery, the rats were anesthetized and dissected. After finding the jugular vein or artery, blood samples were drawn with a syringe and left to rest for $30 \mathrm{~min}$ in a blood collection tube. Plasma was separated by centrifugation $(2,500 \mathrm{x} \mathrm{g}$ for $15 \mathrm{~min})$ and stored at $-80^{\circ} \mathrm{C}$ for further analysis. Detection of TNF- $\alpha$, IL-1 $\beta$ and HMGB1 concentration in plasma was by ELISA.

Histopathological examination. Twenty-four hours after surgery, the left lungs from the different groups of rats were collected and immediately washed twice with phosphate-buffered saline, then modified and fixed in $10 \%$ neutral formalin. The samples were successively dehydrated and paraffin embedded. Tissue sections $(4 \mu \mathrm{m})$ were fixed in ethanol at room temperature, stained with hematoxylin and eosin, and analyzed by light microscopy and photographed.

Calculation of cumulative mortality. Ten animals from the different groups were kept under observation for 7 days after surgery. The natural time of death after surgery was recorded. The survival of rats in each group and the cumulative mortality at 7 day was calculated. 
Table I. Evaluation criterion of sepsis severity for rats after CLP.

\begin{tabular}{lll}
\hline $\begin{array}{l}\text { Observation } \\
\text { index }\end{array}$ & Scoring criteria & $\begin{array}{l}\text { Score } \\
\text { value }\end{array}$ \\
\hline
\end{tabular}

$\begin{array}{ll}\text { General } & \text { Normal food intake and activity } \\ \text { condition } & \text { Eating less, tiredness, reduced activity } \\ & \text { Antifeedant, somnolence, inactive } \\ & \text { Lethargy, lower reaction to stimuli } \\ \text { Bowel } & \text { Ruddy bloom, euperistalsis } \\ \text { condition } & \text { Congestion, mild expansion, } \\ & \text { euperistalsis } \\ & \text { Obfuscation, moderate expansion, } \\ & \text { poor motility } \\ & \text { Purple, high expansion, loss of } \\ & \text { peristalsis }\end{array}$

Ascites 3, clear ascites, no smell 1

condition (ml) 3-6, opacity ascites, no smell 2

$>6$, bloody ascites, micro-odor, $\quad 3$

odorous

$>6$, purulent ascites, severe odor

Cecal ligation

condition

Edema, ischemia

Black, necrosis

White, gangrene

Gangrene, surrounded by a large

amount of pus

Lesion Not wrapped around, no necrosis

encapsulation

or exudation around

Wrapped by greater omentum

and intestine

Not wrapped, obvious necrosis

and exudation around

Pus diffusion, not wrapped around

Liver volume (\%,

$0-20$

compared to the

$20-40$

control group)

$40-60$

$>60$

Pulmonary weight

ratio (wet/dry)

4.5

4.5-5.0

$5.0-5.5$

$>5.5$

2

3

4

1

2

3

4

1

condition

CLP, cecal ligation and puncture.

Statistical analysis. Statistical analyses were performed using SPSS 18.0 software (IBM, Chicago, USA). Data were presented as mean $\pm \mathrm{SD}$. Normal distribution variables among different groups were compared using analysis of variance, non-normal distribution and variables were compared with rank-sum test. Parameters between two of the five groups were compared using an LSD t-test. The cumulative survival rate was calculated using the Kaplan-Meier survival method. Enumeration data were analyzed using the Chi-square test. $\mathrm{P}<0.05$ was considered to indicate statistically significant results.

\section{Results}

Comparison of sepsis severity of rats. The rats in the CLP group gradually appeared less active, were cold, had dull fur, stopped drinking water, and had upright fur. Dyspnea occurred in some rats. In addition, some rats appeared with double-ring eyes, had no resistance to passive supine and became slow to respond. The mortality rate was high in the short term.

The rats in the atorvastatin group appeared anorectic, had vertical hair, and were less active or completely inactive. The rats in the LMWH group behaved similarly to the atorvastatin group.

The rats in the combined group also appeared anorectic, with vertical hair, and were less active. The symptoms were less severe than in the CLP group. The sepsis severity scale in the four groups is shown in Table II.

Comparison of plasma TNF- $\alpha$, IL-1 $\beta$, and HMGBI concentrations in each group. At $0 \mathrm{~h}$, the levels of TNF- $\alpha$, IL-1 $\beta$ and HMGB1 in plasma showed no significant difference in the five groups $(\mathrm{P}>0.05)$. In the sham operation group, the levels of TNF- $\alpha$, IL-1 $\beta$ and HMGB1 in plasma had no significant changes $(\mathrm{P}>0.05)$. Compared to the sham operation group, at 4, 8, 12 and $24 \mathrm{~h}$, the levels of TNF- $\alpha$, IL- $1 \beta$ and HMGB1 in plasma of the CLP group significantly increased $(\mathrm{P}<0.05)$. The concentration of TNF- $\alpha$, IL- $1 \beta$ and HMGB1 reached peak levels at 4,8 and $24 \mathrm{~h}$, respectively. Compared to the CLP group, the levels of TNF- $\alpha$, IL-1 $\beta$ and HMGB1 in the atorvastatin, $\mathrm{LMWH}$, and atorvastatin combined with $\mathrm{LMWH}$ group significantly decreased $(\mathrm{P}<0.05)$. In addition, compared to the atorvastatin and LMWH groups, the TNF- $\alpha$ concentration in plasma at $4 \mathrm{~h}, \mathrm{IL}-1 \beta$ concentration in plasma at $12 \mathrm{~h}$ and HMGB1 concentration in plasma at $24 \mathrm{~h}$ in the atorvastatin combined with LMWH group were significantly reduced $(\mathrm{P}<0.05)$ (Table III).

Pathological changes in the lung under by light microscopy. Lung histology was normal in the sham operation group by light microscopy. In the CLP group, lungs exhibited substantial hemorrhage, inflammatory cell infiltration, obvious consolidation, capillary congestion and thrombus formation. In the atorvastatin and LMWH groups, lungs exhibited alveolar hemorrhage, and more inflammatory cell infiltration and reduction in consolidation compared to the CLP group. In the combined group, lung tissue exhibited partial congestion and thickening, a reduction in interstitial pulmonary edema, capillary congestion, less inflammatory cell infiltration and mild lung consolidation (Figs. 1-5).

Cumulative mortality calculation. Ten animals from the different groups were kept under observation for 7 days after surgery. No deaths occurred in the sham operation animals. The 7-day cumulative mortality in the CLP group was $90 \%$. The 7-day cumulative mortality in the atorvastatin, LMWH and combined groups were 60,60 and $40 \%$, respectively, which was significantly decreased in comparison to the CLP group $(\mathrm{P}<0.05), \chi^{2}=16.174$ and $\mathrm{P}=0.003$ was determined by the log-rank test. The overall difference in the five groups was significant by a value of $\alpha=0.05$ (Fig. 6).

\section{Discussion}

Sepsis is a systemic inflammatory response syndrome (SIRS) to infection. The process involves a series of changes in 
Table II. Comparison of sepsis severity scale in the four groups (mean $\pm \mathrm{SD}$ ).

\begin{tabular}{|c|c|c|c|c|c|c|}
\hline Group & Case (n) & $0 \mathrm{~h}$ & $4 \mathrm{~h}$ & $8 \mathrm{~h}$ & $12 \mathrm{~h}$ & $24 \mathrm{~h}$ \\
\hline Sham operation & 6 & $7.0 \pm 0.5$ & $7.2 \pm 0.5$ & $7.3 \pm 0.6$ & $7.1 \pm 0.5$ & $7.2 \pm 0.6$ \\
\hline CLP & 6 & $7.0 \pm 0.5$ & $12.2 \pm 2.0^{\mathrm{a}}$ & $16.6 \pm 2.5^{\mathrm{a}}$ & $22.8 \pm 3.0^{\mathrm{b}}$ & $27.2 \pm 4.0^{\mathrm{b}}$ \\
\hline Atorvastatin & 6 & $7.0 \pm 0.4$ & $9.0 \pm 1.5$ & $12.2 \pm 2.0^{\mathrm{ac}}$ & $16.3 \pm 2.5^{\mathrm{ac}}$ & $21.2 \pm 2.5^{\mathrm{ac}}$ \\
\hline LMWH & 6 & $7.0 \pm 0.4$ & $10.0 \pm 1.8$ & $11.2 \pm 2.2^{\mathrm{ac}}$ & $17.3 \pm 2.5^{\mathrm{ac}}$ & $20.2 \pm 2.0^{\mathrm{ac}}$ \\
\hline Combined & 6 & $7.0 \pm 0.6$ & $8.4 \pm 1.5^{\mathrm{c}}$ & $10.0 \pm 1.7^{\mathrm{ac}}$ & $12.20 \pm 0.8^{\mathrm{ac}}$ & $16.4 \pm 1.5^{\mathrm{bd}}$ \\
\hline
\end{tabular}

Compared with sham operation group: ${ }^{\mathrm{a}} \mathrm{P}<0.05,{ }^{\mathrm{b}} \mathrm{P}<0.01$; compared with $\mathrm{CLP}$ group: ${ }^{\mathrm{C}} \mathrm{P}<0.05,{ }^{\mathrm{d}} \mathrm{P}<0.01$. CLP, cecal ligation and puncture; LMWH, low-molecular-weight heparin.

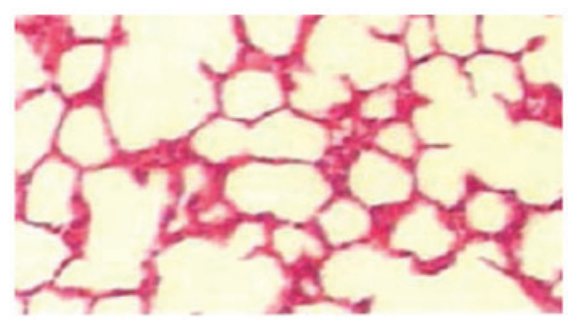

Figure 1. Normal pulmonary pathology group (H\&E; magnification, x200). $\mathrm{H} \& \mathrm{E}$, hematoxylin and eosin.



Figure 2. Pulmonary pathology in the CLP group (H\&E; magnification, $\mathrm{x} 200)$. CLP, cecal ligation and puncture; H\&E, hematoxylin and eosin.

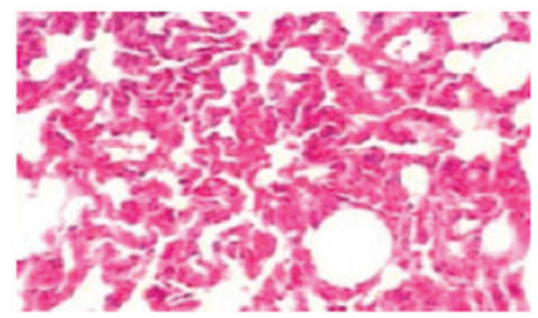

Figure 3. Pulmonary pathology in atorvastatin group (H\&E; magnification, $\mathrm{x} 200)$. H\&E, hematoxylin and eosin.

the body, such as inflammation, cellular immunity, blood coagulation and tissue damage. Mounting evidence indicates that the inflammatory response induced by severe infection is closely related to the changes in coagulation function, and inflammatory reactions, while changes in coagulation function are closely related to the severity and mortality of sepsis (13). At present, the imbalance of coagulation function, inflammatory reactions and immune suppression are considered to comprise the main pathophysiological basis of high incidence and high mortality of sepsis. Inflammatory reactions can activate the

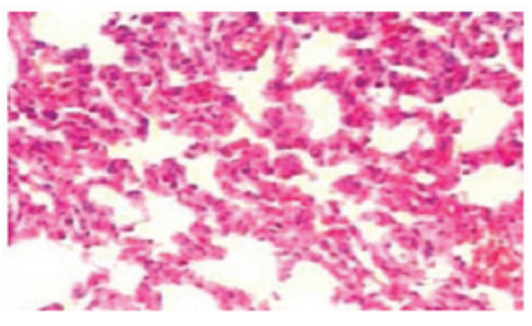

Figure 4. Pulmonary pathology in low-molecular-weight heparin group (H\&E; magnification, x200). LMWH, low-molecular-weight heparin; $\mathrm{H} \& \mathrm{E}$, hematoxylin and eosin.

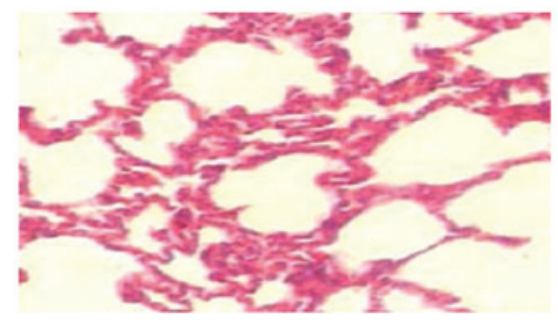

Figure 5. Pulmonary pathology in combined group (H\&E; magnification, $\mathrm{x} 200)$. H\&E, hematoxylin and eosin.

coagulation system. Microthrombosis in the high coagulation state can result in vascular embolism and disseminated intravascular coagulation (DIC), which is the basic induction of severe sepsis and septic shock, thus coagulation disorder is an important part of the pathogenesis of sepsis (14). Although breakthroughs have been made in understanding the pathophysiology of the inflammatory response in recent years and bundle therapies have been used in the treatment of sepsis, the mortality of severe sepsis is $40 \%$, and $>50 \%$ in patients complicated with organ failure (3). Studies suggest that sepsis is characterized by the overexpression of pro-inflammatory cytokines $(15,6-8)$, such as early inflammatory cytokine, TNF- $\alpha$ as well as IL-1 $\beta$, IL-6, IL-8 and HMGB1. Overexpression of inflammatory factors leads to multiple organ dysfunction and increased mortality.

In this study, sepsis was induced by CLP. Bacteria that released into the peritoneal cavity and stimulated monocytes, macrophages and endothelial cells to produce a variety of inflammatory cytokines, leading to metabolic, hormonal and neuroendocrine changes in the body, which resulted in abnormal cell function and organ failure. TNF- $\alpha$ is an impor- 




Figure 6. The cumulative survival rate of rats of each group over 7 days (Kaplan-Meier method). Groups: 1, sham operation group; 2, CLP group; 3 , atorvastatin group; 4, LMWH group; 5, combined group. CLP, cecal ligation and puncture; LMWH, low-molecular-weight heparin.

tant initiator in sepsis. It induces the release of inflammatory mediators, which results in a 'waterfall pattern' cascade reaction. When bacteria enter the body, TNF- $\alpha$ appears early in the circulation and quickly reaches peak levels, inducing a series of inflammatory changes in vascular endothelial cells and the microcirculation (16). The levels of plasma TNF- $\alpha$ in infected animals significantly increased at $1 \mathrm{~h}$ after infection, reached peak levels at $2 \mathrm{~h}$ and returned to control levels at $4 \mathrm{~h}$. The concentration of TNF- $\alpha$ positively correlated with the severity of pathological changes and tissue damage. TNF- $\alpha$ is involved in metabolic processes and immune responses, activates the coagulation process and stimulates macrophages to produce IL-1 $\beta$, IL- 6 as well as other inflammatory factors (17). IL-1 $\beta$ and IL-6 are considered to be closely related to SIRS severity and mortality $(18,19)$. HMGB1 is a late inflammatory mediator, mainly released by monocytes and macrophages under stimulation by infection and trauma, and the release intensity is dose- and time-dependent with TNF- $\alpha$ and IL-1 $\beta$ (20). HMGB1 as a late inflammatory mediator, and has been recognized as a key factor in the lethal effect of sepsis, and its level in vivo will directly affect the severity of the body's response and prognosis of sepsis (21).

Previous findings have indicated that in the process of sepsis, HMGB1 has a role in coagulation and thrombosis, which is consistent with the cross activation of inflammation and coagulation in the process of sepsis (22). It has been reported that HMGBl reaches peak levels at 12-24 h. Compared with early inflammatory mediators including IL-1 $\beta$ and TNF- $\alpha$ which return to normal levels 6-12 h later, the therapeutic time window for HMGBl is relatively larger, thus the targeting therapeutic effect is important (23).

The current results have shown that compared to the sham operation group, at 4, 8, 12 and $24 \mathrm{~h}$, the concentration of inflammatory cytokines in the CLP group had increased significantly, where the TNF- $\alpha$ concentration in plasma peaked at $4 \mathrm{~h}$, the IL-1 $\beta$ concentration in plasma peaked at $8 \mathrm{~h}$, and the HMGB1 concentration in plasma peaked at $24 \mathrm{~h}$. Compared to the CLP group, the concentration of inflammatory cytokines in the atorvastatin, $\mathrm{LMWH}$, and atorvastatin combined with $\mathrm{LMWH}$ 
groups decreased significantly, and there were significant differences in the four groups. Compared to the atorvastatin and LMWH groups, the TNF- $\alpha$ concentration in plasma at $4 \mathrm{~h}$, IL-1 $\beta$ concentration in plasma at $12 \mathrm{~h}$ and HMGB1 concentration in plasma at $24 \mathrm{~h}$ in the atorvastatin combined with LMWH group decreased significantly $(\mathrm{P}<0.05)$. The results showed that atorvastatin and LMWH had a significant inhibitory effect on the release of inflammatory factors, and the two had a synergistic effect.

Statins, 3-hydroxy-3-methyl-glutaryl-CoA reductase inhibitors, possess effects including anti-inflammatory properties, immune regulatory properties, antioxidant and anticoagulant properties as well as can stabilize the endothelial cells of blood vessels. These effects are referred to as the pleiotropic effects of statins (24), which are independent of their lipid lowering effect and, can be used for treatment of sepsis. Clinical studies demonstrated that statins are beneficial for sepsis (25). In basic research, sepsis was induced by CLP in mice, and the average survival rate of mice treated with statins was 4-fold higher than that in the control group (26). Crosstalk exists between inflammatory reactions and coagulation disorders, both of which play important roles in the pathogenesis of sepsis as initiating factors. Thus, the intervention of coagulation disorders may be a new therapeutic area for sepsis treatment (27). Heparin can inhibit HMGBl pro-inflammatory activity by changing the conformation of HMGB1 by combining with 6-12 amino acid residues in its $\mathrm{N}$-terminal region (28-30). LMWH plays a role in alleviating the inflammatory response likely by blocking $\mathrm{NF}-\kappa \mathrm{B}-$ mediated inflammatory effects, which may be one of the mechanisms for improving the prognosis in critically ill patients. LMWH can reduce the APACHE II score in elderly patients with severe pneumonia, shorten the mechanical ventilation time and ICU stay time, thereby improving the prognosis of patients (31). The efficacy and mechanism of combined treatment with the two drugs in sepsis have not been reported. In this study, we found that the sepsis severity scores of rats decreased after atorvastatin or LMWH treatment, and their combination afforded better effects. Our findings also showed that in the sham operation group, the lung tissue was normal by light microscopy. In the CLP group, lungs exhibited substantial alveolar hemorrhage, a large amount of inflammatory cell infiltration, obvious consolidation, capillary congestion and thrombus formation. In the atorvastatin and LMWH heparin groups, lungs exhibited alveolar hemorrhage, inflammatory cell infiltration, and a reduction in consolidation compared to the CLP group. In the combined therapy group, lungs exhibited partial congestion and thickening, a reduction in interstitial pulmonary edema, less capillary congestion, less inflammatory cell infiltration and mild lung consolidation. No deaths occurred in the sham operation animals. The 7-day cumulative mortality in the CLP group was $90 \%$. The 7-day cumulative mortality in the atorvastatin group, LMWH group and combined group were 60,60 and 40\%, respectively, which significantly decreased in comparison to the CLP group.

The results of the present study showed that combined treatment with statins and LMWH reduced sepsis-associated mortality significantly by protecting and improving the functions of tissues and organs at multiple levels by different mechanisms, and exerting anti-inflammatory effects. Lung, as the most involved and important organ in the pathophysiology of sepsis, mainly exhibits alveolar capillary permeability changes and hypoxia (32). The present findings show that the combined treatment of statins and LMWH in lung injury attenuated pathologic destruction, greatly decreased inflammatory cell accumulation and maintained the alveolar structure. These results show that the combined treatment of statins and LMWH can reduce the lung injury in sepsis and that the two have a synergistic effect. A possible mechanism is that atorvastatin and LMWH have inhibitory effects on the expression of early and late inflammatory factors, thereby reducing the inflammatory reaction of rats with sepsis.

In conclusion, combined treatment with atorvastatin and LMWH inhibited the release of inflammatory cytokines, decreased the sepsis severity score, and lowered the mortality rate and the two had a synergistic effect. However, whether the treatment is valid in clinic requires further prospective randomized controlled studies of large sample sizes.

\section{Acknowledgements}

This study was supported by grants from Shandong Provincial Natural Science Foundation, China (no. ZR2013HM062) and the Shandong Provincial Science and Technology Development Plan of Medical and Health (no. 2013WS0110).

\section{References}

1. Sakr Y, Elia C, Mascia L, Barberis B, Cardellino S, Livigni S, Fiore G, Filippini C and Ranieri VM: Epidemiology and outcome of sepsis syndromes in Italian ICUs: a muticentre, observational cohort study in the region of Piedmont. Minerva Anestesiol 79: 993-1002, 2013.

2. Kobashi H, Toshimori J and Yamamoto K: Sepsis-associated liver injury: incidence, classification and the clinical significance. Hepatol Res 43: 255-266, 2013.

3. Diament D, Salomão R, Rigatto O, Gomes B, Silva E, Carvalho NB and Machado FR: Guidelines for the treatment of severe sepsis and septic shock - management of the infectious agent - diagnosis. Rev Bras Ter Intensiva 23: 134-144, 2011 (In Portuguese).

4. Dellinger RP, Levy MM, Rhodes A, Annane D, Gerlach H, Opal SM, Sevransky JE, Sprung CL, Douglas IS, Jaeschke R, et al; Surviving Sepsis Campaign Guidelines Committee including The Pediatric Subgroup: Sepsis Campaign: international guidelines for management of severe sepsis and septic shock, 2012. Intensive Care Med 39: 165-228, 2013.

5. Fukushima H, Nishio K, Asai H, Watanabe T, Seki T, Matsui H, Sugimoto M, Matsumoto M, Fujimura Y and Okuchi K: Ratio of von Willebrand factor propeptide to ADAMTS13 is associated with severity of sepsis. Shock 39: 409-414, 2013.

6. Hatherill M, Tibby SM, Turner C, Ratnavel N and Murdoch IA: Procalcitonin and cytokine levels: relationship to organ failure and mortality in pediatric septic shock. Crit Care Med 28: 2591-2594, 2000.

7. King EG, Bauzá GJ, Mella JR and Remick DG: Pathophysiologic mechanisms in septic shock. Lab Invest 94: 4-12, 2014.

8. Aslani F, Schuppe HC, Guazzone VA, Bhushan S, Wahle E, Lochnit G, Lustig L, Meinhardt A and Fijak M: Targeting high mobility group box protein 1 ameliorates testicular inflammation in experimental autoimmune orchitis. Hum Reprod 30: 417-431, 2015.

9. Subramani J, Kathirvel K, Leo MD, Kuntamallappanavar G, Uttam Singh T and Mishra SK: Atorvastatin restores the impaired vascular endothelium-dependent relaxations mediated by nitric oxide and endothelium-derived hyperpolarizing factors but not hypotension in sepsis. J Cardiovasc Pharmacol 54: 526-534, 2009.

10. Lu X, Zhao L and Xu YH: Low molecular weight heparin prevents CLP-induced acute lung injury in rats by anti-inflammatory coagulation. Bosn J Basic Med Sci 13: 50-56, 2013. 
11. Liu FY and Liu TF: Experimental zoology. Beijing China Science and Technology Press, Beijing, pp209-210, 2005 (In Chinese).

12. Wichterman KA, Baue AE and Chaudry IH: Sepsis and septic shock - a review of laboratory models and a proposal. J Surg Res 29: 189-201, 1980.

13. Levi M, van der Poll T and Schultz M: New insights into pathways that determine the link between infection and thrombosis. Neth J Med 70: 114-120, 2012.

14. Schouten $M$, Wiersinga WJ, Levi $M$ and van der Poll T: Inflammation, endothelium, and coagulation in sepsis. J Leukoc Biol 83: 536-545, 2008.

15. Zhang L and An YZ: The stage-biomarkers of the sepsis. Zhongguo Wei Zhong Bing Ji Jiu Yi Xue 23: 509-512, 2011 (In Chinese)

16. Khalid U, Jenkins RH, Pino-Chavez G, Bowen T, Fraser DJ and Chavez R: A localized ischemic preconditioning regimen increases tumor necrosis factor $\alpha$ expression in a rat model of kidney ischemia-reperfusion injury. Exp Clin Transplant 13: 535-542, 2015

17. Zou JL, Yin ZP, Zhang LQ, Wang YQ and Qi GX: Expression of TNG- $\alpha$ and IL-6 in tissues and serums in the early stage of myocardial ischemia-reperfusion in rat. J China Med Univ 25: 541-543, 2013 (In Chinese).

18. Zhao L, An R, Yang Y, Yang X, Liu H, Yue L, Li X, Lin Y, Reiter RJ and Qu Y: Melatonin alleviates brain injury in mice subjected to cecal ligation and puncture via attenuating inflammation, apoptosis, and oxidative stress: the role of SIRT1 signaling. J Pineal Res 59: 230-239, 2015.

19. Bosmann M, Russkamp NF and Ward PA: Fingerprinting of the TLR4-induced acute inflammatory response. Exp Mol Pathol 93 319-323, 2012.

20. Zhu H, Cai PP, Yin X and Zhu J: Role of high mobility group protein B1 in acute lung injury in rats with sepsis. Chin J Crit Care Med 23: 253-254, 2011 (In Chinese).

21. Ito T, Kawahara K, Nakamura T, Yamada S, Nakamura T, Abeyama K, Hashiguchi T and Maruyama I: High-mobility group box 1 protein promotes development of microvascular thrombosis in rats. J Thromb Haemost 5: 109-116, 2007.

22. Wang $\mathrm{H}$ and Liu D: Baicalin inhibits high-mobility group box 1 release and improves survival in experimental sepsis. Shock 41 : 324-330, 2014.
23. Lee SA, Kwak MS, Kim S and Shin JS: The role of high mobility group box 1 in innate immunity. Yonsei Med J 55: 1165-1176, 2014.

24. Kouroumichakis I, Papanas N, Proikaki S, Zarogoulidis P and Maltezos E: Statins in prevention and treatment of severe sepsis and septic shock. Eur J Intern Med 22: 125-133, 2011.

25. Janda S, Young A, Fitzgerald JM, Etminan M and Swiston J: The effect of statins on mortality from severe infections and sepsis: a systematic review and meta-analysis. J Crit Care 25: 656.e7-22, 2010.

26. Beffa DC, Fischman AJ, Fagan SP, Hamrahi VF, Paul KW, Kaneki M, Yu YM, Tompkins RG and Carter EA: Simvastatin treatment improves survival in a murine model of burn sepsis: role of interleukin 6. Burns 37: 222-226, 2011.

27. Jaimes F, De La Rosa G, Morales C, Fortich F, Arango C, Aguirre D and Muñoz A: Unfractioned heparin for treatment of sepsis: a randomized clinical trial (The HETRASE Study). Crit Care Med 37: 1185-1196, 2009.

28. Zhao D, Ding R, Mao Y, Wang L, Zhang Z and Ma X: Heparin rescues sepsis-associated acute lung injury and lethality through the suppression of inflammatory responses. Inflammation 35: 1825-1832, 2012.

29. Spratte J, Meyer zu Schwabedissen H, Endlich N, Zygmunt M and Fluhr H: Heparin inhibits TNF- $\alpha$ signaling in human endometrial stromal cells by interaction with NF- $\kappa \mathrm{B}$. Mol Hum Reprod 19: 227-236, 2013.

30. Liu ZY, Zhu H and Ma XC: Systematic evaluation of heparin treatment in sepsis. Chin J Crit Care Med 26: 135-141, 2014 (In Chinese)

31. Wang P, Wang X, Zhang LJ, Yang F, Wang GX, Li XL and Huang X: Effect of low molecular weight heparin on the prognosis of elderly patients with severe pneumonia. Chin J Crit Care Med 25: 734-737, 2013 (In Chinese).

32. Gill SE, Taneja R, Rohan M, Wang L and Mehta S: Pulmonary microvascular albumin leak is associated with endothelial cell death in murine sepsis-induced lung injury in vivo. PLoS One 9: e88501, 2014 